\title{
Low Complex Standard Conformable Transceiver based on Doppler Spread for DVB-T2 Systems
}

\author{
Behnam. Akbarian \\ Faculty of Yadegar-e- Imam Khomeini (RAH) Shahr-e- Rey branch, Islamic Azad University, Iran \\ Be.akbarian@yahoo.com \\ Saeed. Ghazi-Maghrebi * \\ Faculty of Yadegar-e- Imam Khomeini (RAH) Shahr-e- Rey branch, Islamic Azad University, Iran \\ S_ghazi2002@yahoo.com
}

Received: 04/Feb/2020 Revised: 24/OCT/2020 Accepted: 28/Nov/2020

\begin{abstract}
This paper addresses a novel Alamouti space-frequency block decoding scheme with discontinuous Doppler diversity (DDoD) and cyclic delay diversity (CDD). We investigate different antenna diversity concepts, which can be applied to orthogonal frequency division multiplexing (OFDM) systems over highly frequency selective channels. The main object of this research is standard compatibility and the effect of simple diversity techniques on the channel fading properties. Therefore, we analyze a receiver in terms of the effective channel transfer function, which leads to the possibility of optimizing diversity. Besides, a novel transceiver using DDoD is proposed, which increases the Doppler spread of the multipath fading channel without causing additional Intercarrier Interference (ICI). Moreover, an efficient Alamouti encoder and decoder based on CDD is proposed, which allows a high reliability and capacity enhancement. In order to evaluate the capability of that, we have implemented this scheme for the second-generation terrestrial video broadcasting (DVB-T2) system over different channels. Furthermore, mathematical analysis and simulation results show the bit error performance of the modified encoding method with these diversity techniques, performs mostly better than the other forms of encoding Alamouti over highly frequency-selective channels such as single frequency networks (SFN). The other advantages of the proposed method are simplicity, flexibility, and standard compatibility.
\end{abstract}

Keywords: SFBC; Transceivers; Diversity; MIMO; OFDM.

\section{1- Introduction}

The orthogonal frequency division multiplexing (OFDM) is known as an attractive modulation scheme for high-rate for transforming a frequency selective channel into multiple flat-fading channels [1], [2]. Moreover, multipleinput multiple-output (MIMO) is a very popular technology to increase communication reliability. Combining OFDM modulation with MIMO technology can be used to overcome the multipath distortion and increasing robustness over wireless fading channels [3]-[7] For this propose, the authors in [8] and [9] surveyed Space-time block codes (STBC) as a suitable multiple antenna technology. However, it needs processing at both transmitter and receiver.

To provide reliable transmission, some technologies like space-time coding are constrained with various designs such as the STBC transmission [10], [11]. Also, the Alamouti method with transmit antenna selection in flat Rayleigh fading channels is investigated in [5], [12], [13]. In [14], an improvement in the Alamouti method has been proposed by the combination of the Alamouti scheme and Bell Laboratories layered space-time (BLAST) methods based on using minimum mean square error (MMSE) detection. However, the results in [14] are valid only when the channel frequency response does not change over the entire Alamouti block code. It has also been shown in [15] that an SFBC-OFDM scheme and a finite-impulse response minimum-mean-square-error (FIR-MMSE) ICI cancellation algorithm can be used together for doublyselective channels. It must be noted that the proposed solutions are not fully satisfactory due to incompatibility and inflexibility with standard systems such as digital system broadcasting. Also, the complexity of the equalizer will be increased to provide the satisfactory performance of the system for highly selective fading channels. In [16], the authors concentrated on turbo equalization receivers for frequency preceded collaborative spatial multiplexing (CSM) MIMO in the uplink of long term evolution advanced (LTE-A) system.

\section{1-1- Motivation and Related Works}

In recent years, different comparisons between theoretical and practical planning methods for DVB-T2 are presented 
in [17]-[20]. For instance, the authors in [17] proposed a new timing detection metric for frequency-selective fading. The symbol timing synchronization scheme was investigated to improve the estimation accuracy. Also, a rotated and cyclic Q-delayed (RCQD) quadrature amplitude modulation was proposed for DVB-T2 systems. Therefore, this system needs a sphere damping algorithm for fading channels, and the sphere-decoder is used for multiple input multiple output detection. As a result, the performance over fading channels can be improved. However, these methods improve the performance of the system with the considerable cost of complexity and time of the process, which makes critical problems for online broadcasting [21], [22]. Generally, the simple Alamouti decoding suffers from the time-variation of the channel, which is not necessarily flat over the entire Alamouti block code [19]. As a result, using the simple Alamouti decoding will cause performance degradation in such channels [23], [24]. Moreover, many multiple antenna technologies require processing at both transmitter and receiver [25], [26]. In practice, the delay diversity (DD) method, which is known as a simple method for increasing diversity, has been attracted as a simple and efficient method in the past few years [27], [28]. Other diversity techniques are cyclic delay diversity (CDD) and Discontinuous Doppler diversity (DDoD) [29], [30]. In fact, the idea of increasing diversity by using multiple transmit (Tx) antennas is not new. However, there is a lack of exploiting temporal diversity for DVB systems [31]. Therefore, the application of DDoD for DVB-T2 becomes attractive. Hence, there is still room for a much better decoding algorithm, increasing diversity, and exploiting standard compatibility methods.

\section{1-2- Contributions and Organization}

In a recent publication [32], we have used the conventional Alamouti scheme with CDD. To combat the performance degradation of the conventional Alamouti scheme, in this paper some methods of Alamouti encoding and decoding using CDD and DDoD are proposed. The other objective of this work is to analyze the performance of the CDD in different forms of Alamouti encoding scheme with two transmit antennas on the DVB-T2 systems [33] in highly frequency-selective channels. Also, in DDoD method, partially shifts each spectrum of an OFDM symbol at the transmitter using different phase shifts in the time domain at different antennas after the OFDM modulation. In this research, we modify the form of Alamouti encoding to obtain a significant performance enhancement in a bit error rate (BER) without increasing the number of antennas. The other advantages of the proposed scheme are its simplicity, flexibility, compatibility, high reliability, and tolerability with respect to the standard Alamouti scheme. This means that a receiver does not need to be aware of their implementations at the transmitter. The experimental results show that the proposed scheme in this paper can obtain a significant performance enhancement in the BER criterion without increasing the number of antennas.

This paper is organized as follows: in Section 2, the model system is presented which are included CDD, DDoD, and standard Alamouti code, channel properties, and determination of cyclic delay value. In Section 3, we present our proposed scheme which is an efficient codingdecoding Alamouti with CDD and DDoD. Section 4 provides simulation results including different delays with respect to two types of delay diversity techniques and presenting the property compatibility of the MIMOOFDM with the CDD and DDoD schemes. In addition, the proposed scheme is compared with other well-known methods. Finally, the conclusion results of this research are presented in Section 5.

\section{2- Model System}

In DVB-T2 systems, in order to enhance bandwidth efficiency, multiple antenna technologies are used [33][36]. In other words, the DVB-T2 system output is typically a single signal to be transmitted. There is assumed that all DVB-T2 receivers shall be able to process the encoding Alamouti signals [37], [38]. Also, we do not require the transmitter to use a different frequency when distributing the same program since the guard interval features [27]. Therefore, we develop a single frequency network (SFN), where all transmitters use identical signals. We will have substantial improvements in the frequency economy and improve coverage for mobile reception due to diversity gain [1]. Figure 1 shows the front end of the DVB-T2 transmitter which is introduced in [33]. The system input may be one or more MPEG-2 transport streams that are built as a frame after, processing, interleaving and modulation. In the next step, after error protection, mapping, and interleaving, the MIMO coding is performed [33]. Then each independent data stream together pilots and transmission parameter signaling (TPS) data are arranged in an OFDM frame. After IFFT transformation, peak to average power ratio (PAPR) reduction, and guard interval (GI) addition, P1 symbol insertion is performed which can be used for signaling and detection purposes.

\section{2-1- Diversity Techniques}

Transmit antenna diversity plays an important role to increase the robustness and reliability of wireless fading channels. The DD scheme is a simple diversity scheme that was proposed by Witteneben in 1993 [28]. The DD scheme 



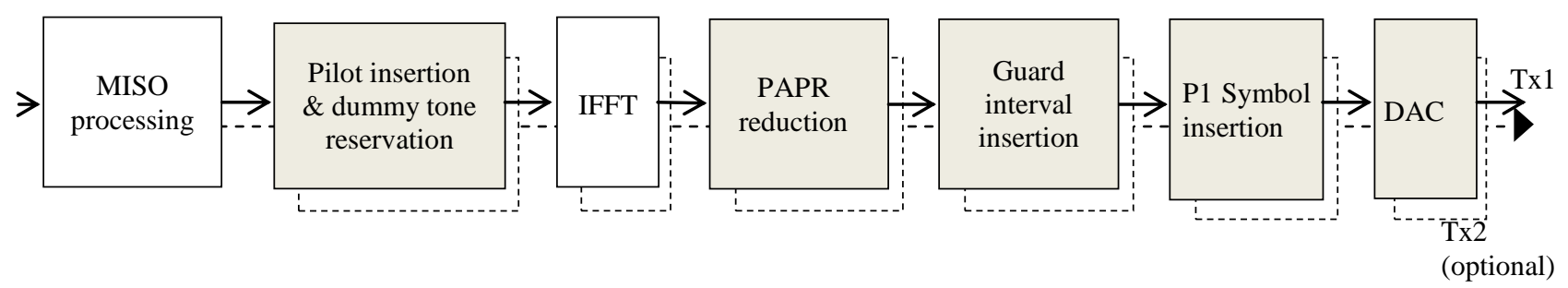

To transmitter(s)

Fig. 1 The front end of the DVB-T2 transmitter [33]

has regained attraction recently as a transmit antenna diversity method for establishing a reliable link in fading channels. In principle, in the DD scheme, delay diversity of a signal over further TX-antenna is transmitted. To avoid ISI, the guard interval length NG should be longer than $L_{m a x}+d_{i}$ (maximum channel delay and delay diversity). The linearity features allow us to implement the DD scheme at the receiver side. However, the channel delay spread increment is imposed on the system. In order to solve this problem, the CDD method is applied in which the TX-antenna specific delays are replaced by cyclic shifts [33]. The frequency selectivity of the channel transfer function can be improved without increasing the observable time-domain channel delay spread at the receiver with the CDD scheme (Table. 1).

In the DDoD method, partially shift each spectrum of an OFDM symbol at the transmitter is applied by using different phase shifts in the time domain at different antennas after the OFDM modulation. In order to perform this operation, signals $\gamma_{i}(m)\left(i=0, . ., N_{T}-1, \mathrm{~m}=-\right.$ $\left.N_{G}, \ldots, N_{F F T}-1\right)$ are determined as sampled exponential function to perform a specific spectral shift of the signal $s(m)$ for specific frequency shift $\left(f_{i}\right)$ and subcarrier spacing $\left(\Delta f_{s c}\right)$. In fact, the main role of Doppler diversity (DoD) is to broaden the Doppler spectrum which results in a decreased channel coherence time such as CDD [39]. According to the results of [39], a specific frequency shift choose based on the value of Doppler width $\left(f_{D}\right)$.

\section{2-2- Standard SFBC Using Alamouti Code}

The Alamouti STBC, which was primarily proposed for the MIMO system contained 2 transmit and 2 receive antennas. Since individual subcarriers can be considered as independent flat fading channels; applying the STBC for OFDM is a straightforward procedure [37], [40], [41].

Two adjacent data-carriers $S_{k}$ and $S_{k+1}^{*}$ for subcarriers $k$ and $k+1$, are considered, that the Alamouti coder outputs two pairs, $\left[S_{k}-S_{k+1}^{*}\right.$ ] for the first antenna, and $\left[\begin{array}{ll}S_{k+1} & S_{k}^{*}\end{array}\right]$ for the second antenna.

\section{3- The Proposed Scheme for Transceivers}

In the latest wireless broadcast systems, such as DVB-T2, in order to increase bandwidth efficiency, multiple antenna technologies are used [21]. The proposed scheme is implemented at both the transmitter and receiver sides, which will be explained in the following.

\section{3-1- Transmitter}

Our proposed scheme, as shown in figure 2, is a genetic MIMO-OFDM based on using the CDD scheme in the DVB-T2 system. The new proposed system provides additional propagation paths and is inserted into the system. Figure 2 shows the Front end of the proposed transmitter side, which has 2 data streams. In the first data stream, after the $N_{F F T}$ point IFFT, a cyclic prefix (CP) is added to each symbol by repeating the end of the same symbol. In this case, the obtained signal $s_{0}(m)$, which is a sequence of $N_{F F T}$ data symbols of $S_{l}$, for $l=0, \ldots, N_{F F T^{-}} l$, is transformed into time-domain and transmitted via the first TX-antenna without delay. In the other data stream, for providing additional diversity and as a result, improvement of system performance, the CDD scheme is implemented.

The average transmission power is kept normalized and independent of the number of related TX-antennas $\left(N_{T}\right)$ by $\left(N_{T}-1\right)^{0.5}$ factor. Based on the block diagram, before inserting the $\mathrm{CP}$ with the length of $N_{G}$, the symbol is shifted cyclically and then the CP is inserted. Therefore, results in the antenna specific TX-signal can be express as bellow:

$$
\begin{aligned}
& s_{\mathrm{i}}(m)=\frac{1}{\left(N_{T}-1\right)^{0.5}} \tilde{s}\left(m-d_{i}^{c y c} \bmod N_{F F T}\right) \\
& \text { for } \quad i=0, \ldots, N_{T}-1, m=-N_{G}, \ldots, N_{F F T}-1
\end{aligned}
$$

where $k$ and $i$ denote the numbers of data symbol and TX-antenna, respectively. 
Table 1: Simple diversity techniques

\begin{tabular}{|c|c|c|c|c|}
\hline $\begin{array}{c}\text { Diversity } \\
\text { Technique }\end{array}$ & Key technology & Condition (to avoid ISI) & More information & Ref. \\
\hline $\begin{array}{l}\text { Delay Diversity } \\
\text { (DD) }\end{array}$ & $\begin{array}{c}\text { The simple method to increase diversity. } \\
\text { (delay diversity schemes are based on } \\
\text { signal shifts } \\
\text { in the time domain,) }\end{array}$ & $N_{G} \geq L_{\max } d_{i \max }$ & $\begin{array}{l}\text { causes the channel delay } \\
\text { spread increment }\end{array}$ & $\begin{array}{l}{[8]} \\
{[9]}\end{array}$ \\
\hline $\begin{array}{l}\text { Cyclic Delay } \\
\text { Diversity (CDD) }\end{array}$ & $\begin{array}{l}\text { CDD scheme enhances the frequency } \\
\text { selectivity of the channel transfer function }\end{array}$ & $N_{G} \geq L_{\max }$ & $\begin{array}{l}\text { Involved channel } \\
\text { estimation }\end{array}$ & $\begin{array}{l}{[19],} \\
{[22]}\end{array}$ \\
\hline $\begin{array}{l}\text { Doppler Diversity } \\
\text { (DoD) }\end{array}$ & $\begin{array}{l}\text { Shift the antenna specific signals in the } \\
\text { frequency domain. }\end{array}$ & $\begin{array}{c}\gamma_{i}(m)=e^{j 2 \pi \cdot f i \cdot T \cdot m} \\
T=1 /\left(N_{F F T} \cdot \Delta f S c\right)\end{array}$ & $\begin{array}{l}\text { Increasing the Doppler with } \\
\text { DoD in OFDM systems in } \\
\text { principle enlarges ICI }\end{array}$ & {$[23]$} \\
\hline $\begin{array}{l}\text { Discontinuous } \\
\text { Doppler Diversity } \\
\text { (DDoD) }\end{array}$ & $\begin{array}{l}\text { The DoD Signal is constant for the } \\
\text { duration of an OFDM symbol. }\end{array}$ & $\begin{array}{c}\gamma_{i}(m)= \\
\left.e^{j 2 \pi \cdot f i \cdot T \cdot(N}{ }_{\text {OFDM }}\left(m \div{ }_{\text {OFDM }}\right)\right)\end{array}$ & Needs further investigation & $\begin{array}{l}{[24]} \\
{[30]}\end{array}$ \\
\hline
\end{tabular}
the signal is shifted cyclically by $d_{i}^{c y c}$ before the CP is added.

After removing the $\mathrm{CP}$ at the receiver side, as long as TX$$
\text { antenna certain delays, } d_{i} \text {, is equal to cyclic shifts, } d_{i}^{c y c}, \mathrm{~b}
$$
oth CDD and DD schemes yield the same signal. The OF DM symbols of the DD signal partly overlap with the CP of the subsequent OFDM symbol at about delay which $\mathrm{i}$ $\mathrm{s}$ a restriction in the choice of $d_{i}$. Therefore, the ISI part o $\mathrm{f}$ the received signal at $k^{\text {th }}$ subcarrier can also be expresse d as:

$$
\begin{aligned}
& R_{I S I}(k)=\frac{1}{\sqrt{N_{F F T}}} \\
& \sum_{l=N_{G}+1}^{L_{\max }+d_{\max }} h(l) \sum_{k=0}^{l-N_{G}} s(m-l) \cdot e^{j 2 \pi k m / N_{F F T}}
\end{aligned}
$$

where $h_{i}(l)$ is the channel impulse response from TXantenna $i$ to the RX-antenna with delays of $l>N_{G}$ samples and the signal part $s(k), k<-N_{G}$, since these terms cause ISI [30]. Also, the minimum length of CP should be $N_{G} \geq L_{\max }$ in the system based on the CDD and this length does not depend on the cyclic delays, $d_{i}^{c y c}$. Therefore, a shorter CP can be chosen and there is an advantage of CDD with respect to the DD that the CDD scheme does not depend on the number of TX-antenna and there is no overlapping of OFDM symbols in it. However, in the case of free-ISI, the DD scheme performs the same as CDD.

To avoid ISI interference, the guard interval length $N_{G} \mathrm{~m}$ ust be $N_{G}>L_{\text {max }}+\max D_{i}$ for $i=0, \ldots, N_{T-1}$, where $L_{\text {max }}$ is $\mathrm{t}$ he maximum channel delay samples. Also, we can choose the maximum possible cyclic delays according to: and

$$
d_{i}{ }^{c y c}=N_{G}+1+d_{i-1}^{c y c}
$$

where $N_{T}$ and $N_{F F T}$ denote the number of TX-antenna and data symbol respectively [30].

\section{3-2- Receiver}

In the DVB-T2 system, standard Alamouti code is used in the transmitter for enhancing spectral efficiency and link reliability. This is done for maximizing the diversity in the receiver while using low complexity equalization based on the maximal ratio combining (MRC) method [10].

In this paper, we have utilized the Alamouti code, which was primarily introduced for MIMO systems with two transmit and two receive antennas. Assume $S_{k}, S_{k+1}$ are the two successive subcarriers, $\left[S_{k},-S_{k+1}{ }^{*}\right]$ and $\left[S_{k+1}, S_{k}{ }^{*}\right]$ are two pairs of MIMO encoder outputs for the first and the second data streams, respectively. On the receiver side, we use a decoding method [1], which is called " $\Delta \mathrm{h}$ Alamouti decoding" in this paper. In this method, the first MIMO-OFDM symbol in (2) is the $S_{0}(k)$, which is transmitted as an un-shifted signal $\left(d_{0}^{c y c}=0\right)$ over the first TX-antenna. The other TX-antenna signal is shifted cyclically by $d_{i}^{c y c}$ before adding the $\mathrm{CP}$ as shown in Figure 2. On the receiver side, after removing the $\mathrm{CP}$, the remaining OFDM time domain symbol is transformed into the frequency domain by an FFT in the receiver side, as shown in Figure 3. The received data can be written in a compact form as follow:

$$
\mathbf{Y}=\mathbf{S} \widetilde{\mathbf{H}}+\mathbf{N}
$$




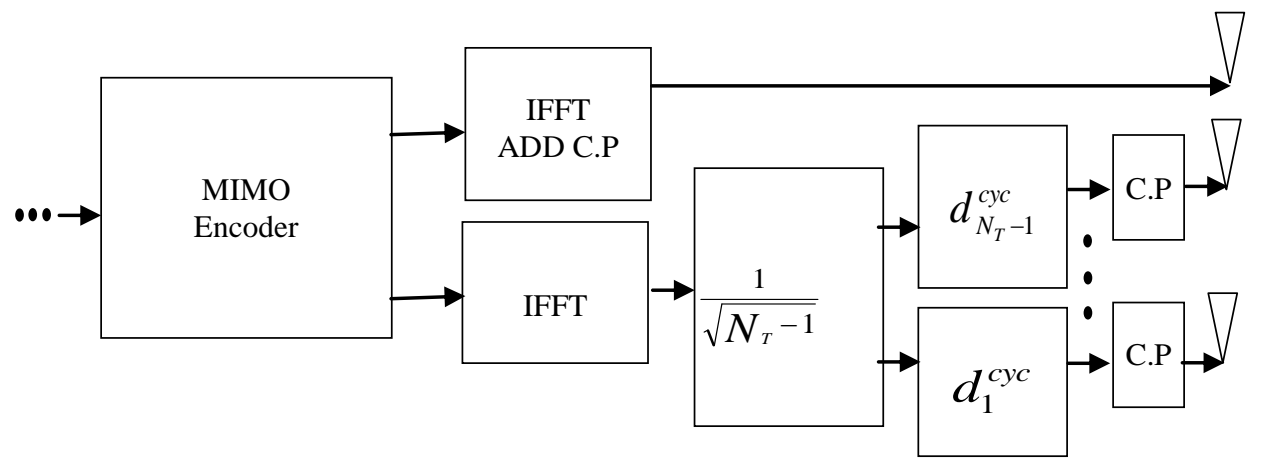

Fig. 2 The front end of the proposed scheme using $\mathrm{CDD}$ at the transmitter side

where $\tilde{\mathbf{H}}$ denotes an equivalent channel transfer function and is defined as bellow:

$$
\begin{aligned}
& \widetilde{\mathbf{H}}=\frac{1}{\sqrt{N_{T}}} \\
& \sum_{i=0}^{N_{T}-1} \sum_{m=0}^{N_{F F T}-1} h_{i}(\mathrm{~m}) \mathrm{e}^{\frac{\mathrm{j} 2 \pi \cdot \mathrm{mk}}{N_{F F T}}} \mathrm{e}^{\frac{-\mathrm{j} 2 \pi \cdot \mathrm{d}_{i}^{\mathrm{gec}} \cdot k}{N_{F F T}}}+\mathbf{N}
\end{aligned}
$$

where $\mathbf{N}$ is a complex Gaussian noise, which is mutually uncorrelated between different subcarriers, $h_{i}$ is the $i^{\text {th }}$ tap of the channel impulse response [30].

Equation (6) means that a receiver cannot distinguish whether a propagation path results from the CDD scheme or the channel itself [4]. After FFT transformation and pilot extraction at the receiver side, we have the following equations:

$$
\left\{\begin{array}{l}
Y_{1}^{K}=S_{k} H_{1,1}^{k}-S_{k+1}{ }^{*} H_{1,2}^{k}+N_{1}^{k} \\
Y_{2}^{K}=S_{k} H_{2,1}^{k}-S_{k+1}{ }^{*} H_{2,2}^{k}+N_{2}^{k} \\
Y_{1}^{K+1}=S_{k+1} H_{1,1}^{k+1}-S_{k}^{*} H_{1,2}^{k+1}+N_{1}^{k+1} \\
Y_{2}^{K+1}=S_{k+1} H_{2,1}^{k+1}-S_{k}^{*} H_{2,2}^{k+1}+N_{2}^{k+1}
\end{array}\right.
$$

where $\tilde{H}_{i, j}^{k}$ is the channel frequency response at carrier $k^{\text {th }}$, between $i^{\text {th }}$ receive antenna and $j^{\text {th }}$ transmit antenna, $N_{i}^{k}$ is AWGN noise at the $k^{\text {th }}$ carrier of $i^{\text {th }}$ receive antenna, and $*$ denotes conjugate operator.

In the standard Alamouti decoding should be assumed that the channel frequency response is constant over two consecutive carriers, i.e. $\tilde{H}_{i, j}^{k}=\tilde{H}^{k+1}{ }_{i, j}$. However, the performance of the standard Alamouti decoding will be degraded over highly frequency-selective channels.

Since there should be quasi-static fading for adjacent subcarriers. In order to overcome this problem, an efficient Alamouti decoding scheme using the CDD scheme is proposed. By considering the changes in the channel matrix over adjacent OFDM carriers, we can write:

$$
\Delta \mathbf{H}^{\mathrm{T}}=\left[\begin{array}{cccc}
0 & 0 & \left(H_{1,2}^{k+1}-H_{1,2}^{k}\right)^{*} & \left(H_{2,2}^{k+1}-H_{2,2}^{k}\right)^{*} \\
0 & 0 & -\left(H_{1,1}^{k+1}-H_{1,1}^{k}\right)^{*} & -\left(H_{2,1}^{k+1}-H_{2,1}^{k}\right)^{*}
\end{array}\right]
$$

where $T$ refers to conjugate transpose operation. Equation (8) confirms that the variation of two consecutive carriers is considered. In other words, if the difference between two consecutive carriers is 0 , the channel frequency response is flat over the Alamouti block. Therefore, there is not any necessity to consider the channel constant over

two consecutive subcarriers. In multiuser detection, optimal error-rate performance is achieved by the joint Maximum Likelihood (ML) detector, which its computational complexity increases exponentially. However, for the last part of detection, we use the results of [42] and [1], which have lower complexity than ordinary methods. For recovering the signal at the receiver side, $\Delta H$ is applied in a detection scheme by the MMSE criterion. Therefore, the estimated transmitted symbol can be expressed as [1]:

$$
\widetilde{\mathbf{S}}=\frac{1}{\operatorname{det}\left(\mathbf{I}_{2}+\mathbf{B} \Delta \mathbf{H}\right)} \operatorname{adj}\left(\mathbf{I}_{2}+\mathbf{B} \Delta \mathbf{H}\right) \cdot \mathbf{B} . \mathbf{Y}
$$

where,

$$
\mathbf{B}=\left(\mathbf{H}^{T} \mathbf{H}\right)^{-\mathbf{1}} \mathbf{H}^{T}
$$

where det and adj denote determinant and adjoint operations, respectively and $\mathbf{I}_{2}$ is the identity matrix of order two.

The transmitted data can be recovered at the receiver side using Eq. (9). It is noticeable that the new scheme has a lower complexity since Eq. (9) does not consist of the 
direct inverse of the matrix $\left(\mathbf{I}_{2}+\mathbf{B} \Delta \mathbf{H}\right)$

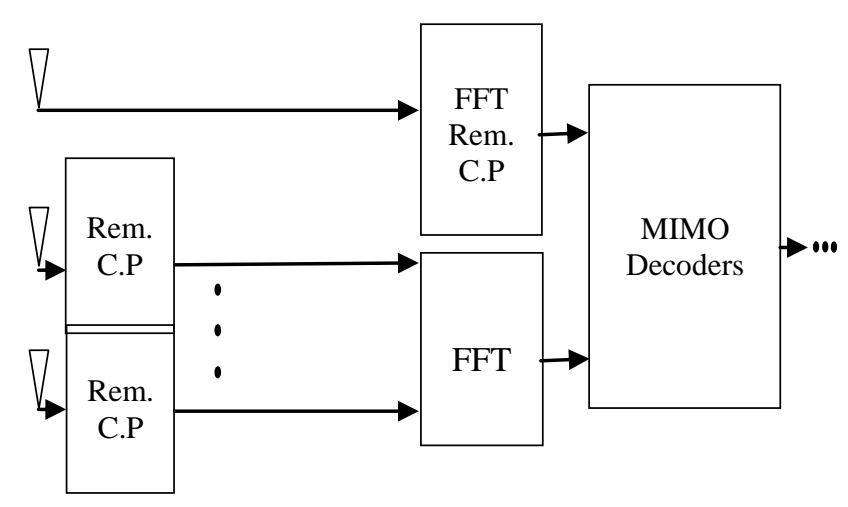

Fig. 3 The proposed scheme using CDD at the receiver side

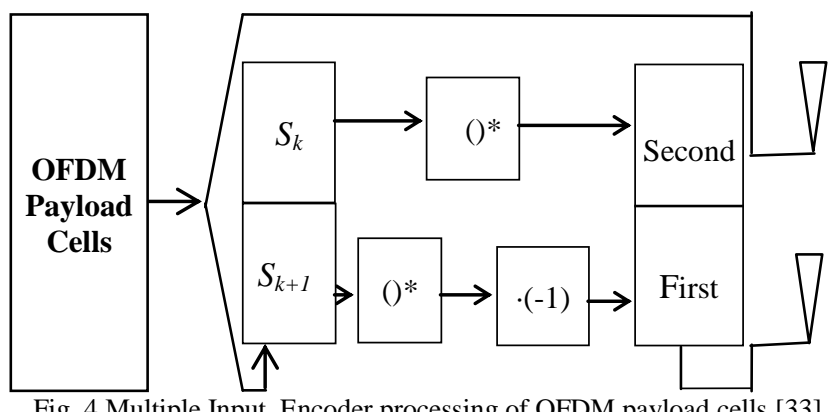

Fig. 4 Multiple Input, Encoder processing of OFDM payload cells [33]

\section{3-3- An Efficient Alamouti coding and decoding using CDD}

An optional initial stage, known as MISO processing in the DVB-T2 standard, provides the initial frequency domain coefficient, which is processed by a modified Alamouti [33]. Then, the DVB-T2 signal to be split between two groups of transmitters on the same frequency without the interference of signals [17].

Figure 4 shows that the MISO processing for transmitters in the MISO group1 copies the unmodified input cells to the output. Also, $N$ data will be an even number, even in the frame closing symbol, even though value CFC (number of active cells in one frame closing symbol) might not be even. Hence we can use it in the proposed strategies.

In this section, we investigated $\Delta \mathrm{h}$-Alamouti decoding using CDD. In other words, we have proposed an efficient scheme, which exploits the modified Alamouti using CDD. On the other hand, the core idea concerns the improvement of the transmitter and receiver sides. On the transmitter side, we use the CDD as an antenna diversity technique and improved Alamouti encoding (Fig. 3). The DVB-T2 standard using a modified form of
Alamouti encoding, which is the respective payload cells are processed in the frequency direction.

In the MIMO encoder module, $\left[S_{k}, S_{k+1}\right]$ is transmitted as the first data stream; without any modification regarding frequency order. In the second data stream, we have pairwise modification $\left[S_{k+1}{ }^{*}, S_{k}{ }^{*}\right]$. At the receiver side, the MIMO decoder module consists of MMSE criterion and a channel matrix equation as bellow:

$$
\begin{aligned}
& \Delta \mathbf{H}^{\mathrm{T}}{ }_{\text {Efficent }}= \\
& {\left[\begin{array}{cccc}
0 & 0 & 0 & 0 \\
\left(H_{1,2}^{k+1}-H_{1,2}^{k}\right)^{*} & \left(H_{2,2}^{k+1}-H_{2,2}^{k}\right)^{*} & -\left(H_{1,1}^{k+1}-H_{1,1}^{k}\right)^{*} & -\left(H_{2,1}^{k+1}-H_{2,1}^{k}\right)^{*}
\end{array}\right]}
\end{aligned}
$$

Then, by applying equations (9) and (10), when the MIMO channel is estimated using the known pilot, the receiver can recover the transmitted data.

\section{3-4- An Efficient Alamouti coding using DDoD}

In this section, we investigate the use of Doppler diversity (DoD), which schemes shift the antennaspecific signals in the frequency domain [43], [44]. This type of scheme is used in studies such as [31]. Figure 5 shows the front end of the proposed scheme using 
Doppler diversity at the transmitter side. After OFDM modulation using an IFFT and guard interval insertion as a cyclic prefix, the signal $s(m)$ is split (copied) into $N_{T}$
TX-branches. After normalization by the factor, the time domain signal in each TX-branch is multiplied by a TXantenna specific signal

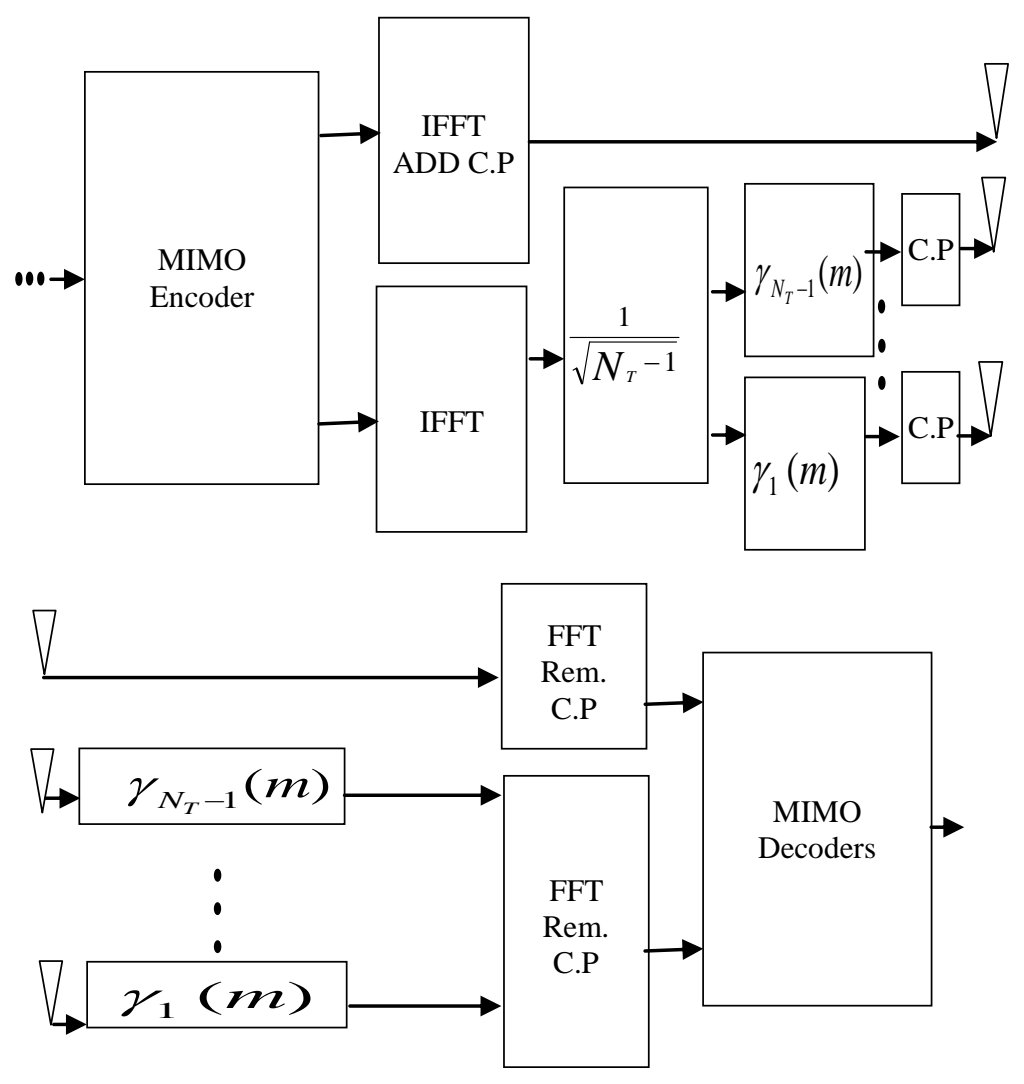

Fig. 5. The proposed scheme using DDoD (transmitter and receiver)

$\gamma_{i}(m)$. Therefore, results in the TX-antenna specific transmission signals can be expressed as [44] :

$$
\begin{aligned}
& s_{i}(m)=\frac{1}{\sqrt{N_{T}}} s(m) .-\gamma_{i}(m) \\
& \text { for } \quad \begin{aligned}
i & =0, \ldots, N_{T}-1 \\
& m=-N_{G}, \ldots, N_{F F T}-1
\end{aligned}
\end{aligned}
$$

According to Fig. 5 (receiver side), the time domain signal for $n^{\text {th }}$ OFDM symbol after removal guard interval can be written as follows [31]:

$$
\begin{aligned}
& r_{n}(m)=\frac{1}{\sqrt{N_{T}}} \\
& \sum_{i=0}^{N_{T}-1 N_{\max }} \sum_{l=0} s_{n}(m-l) h_{i}\left(m+n \cdot N_{\text {OFDM }}, l\right) \cdot \gamma_{i}\left(m+n \cdot N_{\text {OFDM }}\right)
\end{aligned}
$$

where $h_{\mathrm{i}}\left(m+n \cdot N_{\mathrm{FFT}}, 1\right)$ denotes the time-variant Rayleigh fading process with a delay of $l$ samples observed from Tx antenna $i$. After that, the received time-domain signal is transformed into the frequency domain and this processing can be described by the following equation [31]:

$$
\begin{aligned}
& R_{n}(k)=\frac{1}{\sqrt{N}} \sum_{m=0}^{N-1} r_{n}(m) \cdot e^{-j \frac{2 \pi}{N} \cdot k \cdot m}= \\
& \frac{1}{\sqrt{N \cdot N_{T}}} \sum_{q=0}^{N} S_{n}(q) \cdot \sum_{i=0}^{N_{T}} \sum_{l=0}^{N_{\max }} \bar{\gamma}_{i \cdot n} \cdot H_{i, l, n}(q) \cdot e^{-j \frac{2 \pi}{N} \cdot l \cdot q}
\end{aligned}
$$

Where $S(q)$ denotes the DFT of $s(m)$ and,

$$
H_{i, l, n}(q)=\frac{1}{\sqrt{N}} \cdot \sum_{m=0}^{N-1} h_{i, n}(m, l) \cdot e^{-j \frac{2 \pi}{N} k \cdot q}(15)
$$

Equation (14) confirms that the effects of DDoD can be assigned to the channel. Also, this simplifies the parallel transmission (ICI-free) over subcarriers if there are constants channel fading $h_{i}(m, l)$ for the duration of an OFDM symbol. Otherwise, we mitigate the system performance degradation by using the proposed method 
i.e. $\Delta$ h-Alamouti in the third strategy. Therefore, we witness a considerable performance improvement. at the receiver side, and as a result, the transmitted data can be recovered at the receiver side using Eq. (9).

In table 1, the signals $\gamma i(m)$ for DDoD is mentioned. In or der to achieve a TX-antenna specific spectral, these signa ls are chosen as exponential functions shift of the signal $s$ $(m)$, before adding the cyclic prefix.

However, the effects of DDoD can be assigned to the channel. These effects are shown in Fig. 6. We applied DDoD with different $f_{D} / f_{S C}$ to the MISO systems $(2 \times 1)$. We used the $2 \mathrm{k}$ mode (2048 points IFFT) with 4-QAM modulation. The guard interval length is $N_{G}=1 / 4$. For our investigations, we used 9-path multipath Rayleigh channel models which is similar to indoor commercialchannel B models in large open centers, such as shopping malls and airports. Its power-delay profile is [0

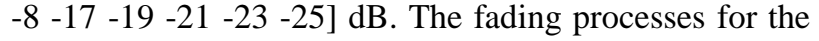
several propagation paths are statistically independent. This result of simulation and mathematical analyses in [23] could be considered to provide design criteria for the choice of TX-antenna parameters for DDoD.

\section{4- Simulation Results}

In this Section, three strategies have been performed to demonstrate the advantages of our proposed scheme. A $\Delta \mathrm{h}$-Alamouti decoding scheme introduced in [1] is used in this simulation. According to [25], the performance of the DD and CDD schemes is equal if the transmission is ISI free. However, by increasing the CP length using the MISO-DD method, noticeable performance degradation will occur at the receiver side. There is a fact to take into account that if the CP length is increased for the DD scheme, the amount of interference will be huge. It should be mentioned that the channel estimation is assumed to be perfect. Also, for better comparison, Table 2 list the parameters and reason for its selection.

\section{4-1- The $\Delta$ h-Alamouti Decoding using CDD (strategy 1)}

We have set up the first strategy to verify the better performance of the proposed $\Delta \mathrm{h}$-Alamouti decoder using the CDD scheme with respect to $\Delta \mathrm{h}$-Alamouti. In the first strategy, the MIMO-OFDM system has been applied to the DVB-T2 standard, in which 8k modes, i.e. 8192 sub-carriers with 4-QAM modulation, are used. We have applied 10000 OFDM symbols with a CP length of $1 / 4$ to a $2 \times 2$ MIMO DVB-T2 system. We have used the P1 multipath Rayleigh fading channel model [6], which has 20 taps without any Doppler effects. In this simulation, $d_{i}^{c y c}=2$, this is because the SNR does not increase further when $d_{i}^{c y c}>1.5 \mu \mathrm{s}$, as shown in [4]. The performance of the un-coded strategy is also evaluated; thus the coding and interleaving blocks are bypassed in our system simulation. Four different profiles are used as MIMO channels with identity power and different phases. In order to make the uncorrelated channel, which is applied in [1], phases are randomly chosen between 0 and $2 \pi$.

In order to simulate highly frequency selective channels, random phase with different values for $a$ and $b$ parameters are used, where $\mathrm{a}$ and $\mathrm{b}$ are the power of the delayed channel and the delayed spread, respectively [26]. In principle, increment in the delay spread and the power of the delay channel convert the channel to a highly frequency-selective channel [1]. Figure 7 shows the results of different methods consisting of the efficient Alamouti decoding scheme using the CDD scheme, $\Delta \mathrm{h}$ Alamouti, and the standard one, when, channel estimation is assumed to be perfect.

From the simulation result, it can be observed that for $a=0.5$, the proposed scheme performs approximately the same as the $\Delta \mathrm{h}$-Alamouti scheme. However, the performance of the standard decoder degrades. Also, for $a=0.9$, the performance of our proposed scheme is better than the $\Delta \mathrm{h}$-Alamouti scheme.

Table 2:Parameters for simulations

\begin{tabular}{|c|c|c|c|}
\hline Fig & Parameter & describe & References \\
\hline 6,10 & $\begin{array}{l}\text { Indoor commercial- } \\
\text { channel B models }\end{array}$ & $\begin{array}{l}N_{\text {tap }}=20 \\
\text { Channel } \\
\text { tap } \\
\text { number } \\
\text { length }\end{array}$ & [6] \\
\hline 10 & $\begin{array}{l}\text { 2k mode (2048 points } \\
\text { IFFT) with 4-QAM } \\
\text { modulation. The guard } \\
\text { interval length is NG } \\
=1 / 4\end{array}$ & $\begin{array}{c}\text { Mode } \\
\text { selection }\end{array}$ & $\begin{array}{c}\text { DVBT-2 } \\
\text { standards } \\
\text { model } \\
{[33]}\end{array}$ \\
\hline $\begin{array}{c}6-9 \\
11-13\end{array}$ & $\begin{array}{c}\text { 8k modes, i.e. } 8192 \\
\text { sub-carriers with 4- } \\
\text { QAM modulation } \\
\quad \mathrm{Nfft}=8192\end{array}$ & FFT size & {$[33],[6]$} \\
\hline
\end{tabular}




\begin{tabular}{|c|c|c|c|}
\hline $6-13$ & $\mathrm{nRX}=2 ; \mathrm{nTX}=2 ;$ & $\begin{array}{c}\text { Number } \\
\text { of } \\
\text { antennas }\end{array}$ & $\begin{array}{c}\text { DVBT-2 } \\
\text { standards } \\
\text { model } \\
{[33]}\end{array}$ \\
\hline $\begin{array}{c}6-11- \\
13\end{array}$ & $\mathrm{~d}_{\mathrm{i}}^{\text {cyc }}=2$ & $\begin{array}{c}\text { Delay } \\
\text { (CDD) }\end{array}$ & {$[4]$} \\
\hline $7-9$, & $\begin{array}{c}\text { DVBT-2 channel } \\
\text { model } \\
11-13\end{array}$ & $\begin{array}{c}\text { the power } \\
\text { of the } \\
\text { delayed } \\
\text { channel } \\
\text { and the } \\
\text { delayed } \\
\text { spread }\end{array}$ & $\begin{array}{c}\text { DVBT-2 } \\
\text { standards } \\
\text { model } \\
{[33] \text { and }} \\
{[1]}\end{array}$ \\
\hline
\end{tabular}

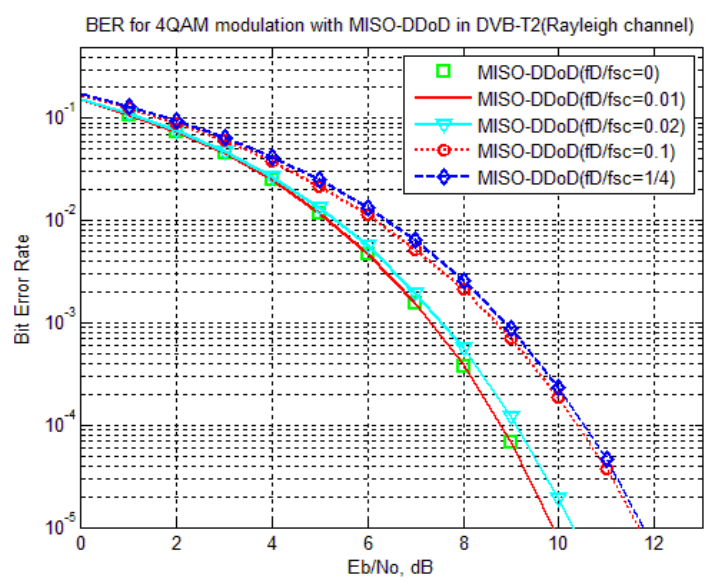

Fig. 6 The effect of discontinues Doppler diversity delay

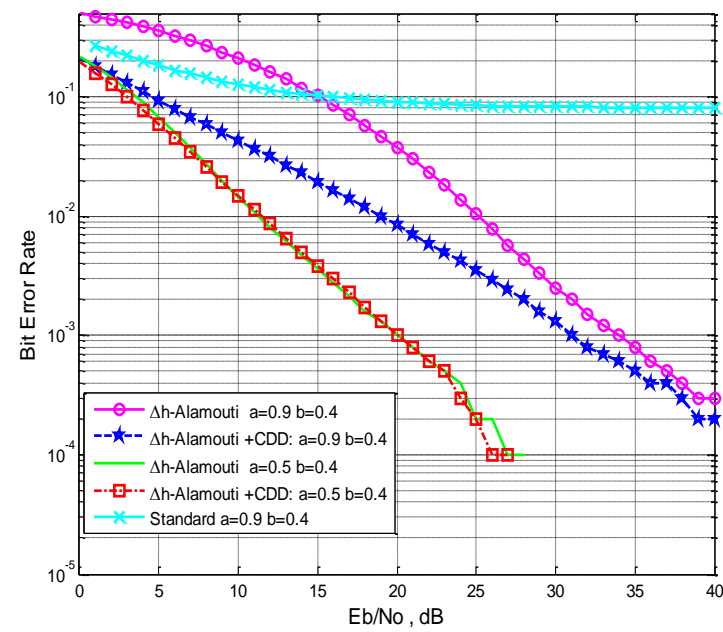

Fig.7 The performance of $\triangle \mathrm{h}$-Alamouti using CDD schemes in BER vs. SNR.

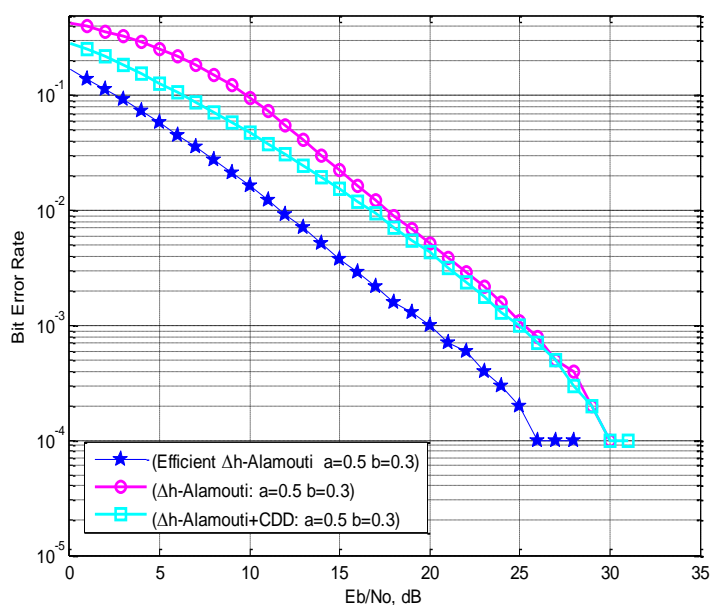

Fig. 8 A comparison of different schemes $(a=0.5, b=0.3)$ in BER vs. SNR.

This improvement is due to the increased degree of diversity of the proposed method, which allows a significant performance enhancement that can be achieved without increasing the number of antennas. Besides, BER is also decreased by using this scheme and the performance of the proposed scheme is still much better than the other schemes. This is because the CDD scheme is capable of offering a larger degree of diversity to improve the performance, which is achieved through the reception of data signals from more than one path. For instance, Figure 7 shows that this scheme outperforms the scheme in [1] by $3.2 \mathrm{~dB}$ at an average BER of 10e-3.

\section{4-2- The Efficient $\Delta$ h-Alamouti Coding and Decoding Using CDD (strategy 2)}

In this section, we investigate the efficient $\Delta \mathrm{h}$-Alamouti coding and decoding using CDD, which is introduced in section 3. We consider the MIMO-OFDM system based on the DVB-T2 standard with 8k mode (8192 subcarriers with 4-QAM modulation) and similar conditions of the previous strategy. The three schemes are compared in different channels.

In Figure 8, we observe that when $a=0.5$, two strategies of $\Delta \mathrm{h}$-Alamouti with and without CDD perform a little convergence in the high SNR. However, the efficient $\Delta \mathrm{h}$ Alamouti coding and decoding using CDD are much better than the other schemes. For instance, at BER= 10e-3, the efficient $\Delta \mathrm{h}$-Alamouti coding and decoding using CDD get about $5 \mathrm{~dB}$ gain than the other schemes.

For relatively high values of $a=0.9$ and $b=0.4$, the performance of the efficient $\Delta \mathrm{h}$-Alamouti coding and decoding using CDD outperforms the previous schemes (Fig. 9). For example, at BER 10e-3, the modified encoding form with CDD gets about $9.8 \mathrm{~dB}$ gain than the 
standard encoding form with CDD. Due to the fact that, when channels are highly frequency-selective (with decreasing $a$ and $b$ ), the efficient $\Delta$ h-Alamouti coding and decoding using CDD can conserve its performance. But, the method of $\Delta$ h-Alamouti with CDD has a minor performance improvement. In the proposed scheme, allows the high tolerability of the system to highly selective channels. Therefore, we gain better performance by using a new modified version of Alamouti encoding with CDD. Because, the efficient $\Delta \mathrm{h}-$ Alamouti coding using CDD provides additional diversity in channels and therefore, improves the system performance.

\section{4-3- The Efficient $\Delta \mathrm{h}$-Alamouti Decoding Using DDoD (strategy 3)}

For an investigation of DDoD, we applied Alamouti

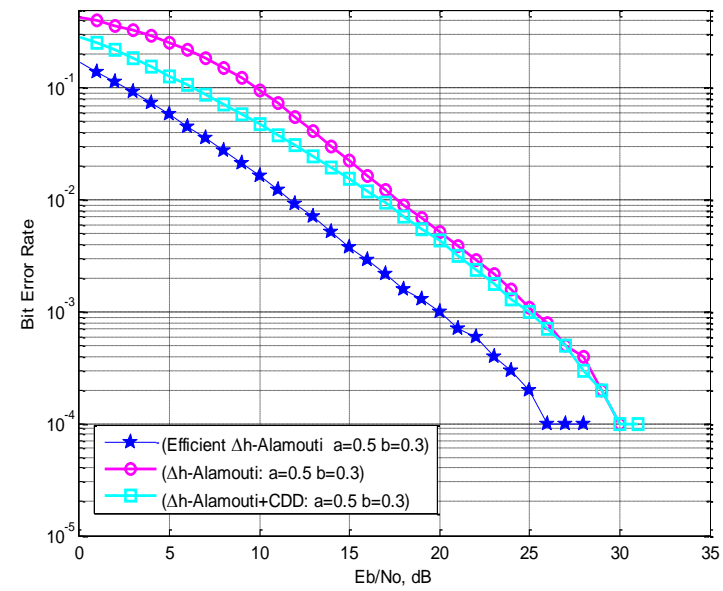

Fig. 8 A comparison of different schemes $(a=0.5, b=0.3)$ in BER vs. SNR.

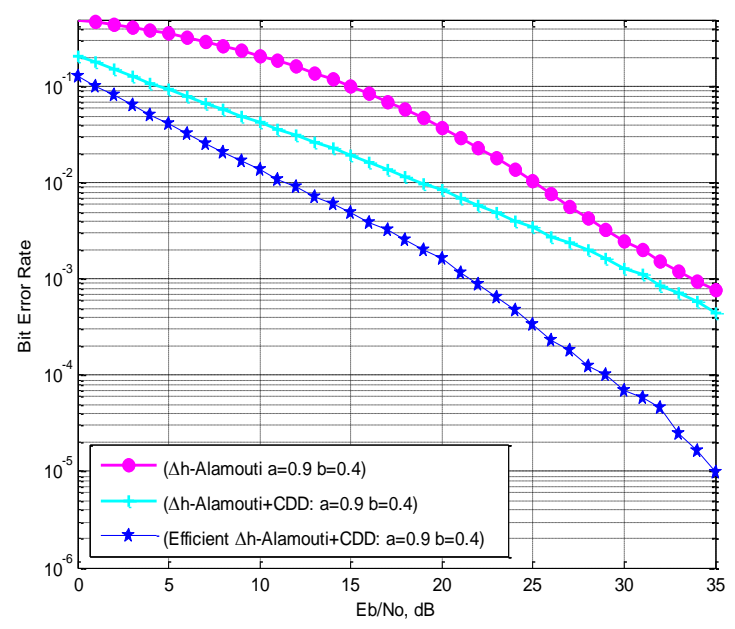

Fig. 9 A comparison of different schemes $(a=0.9, b=0.4)$ in BER vs. SNR.

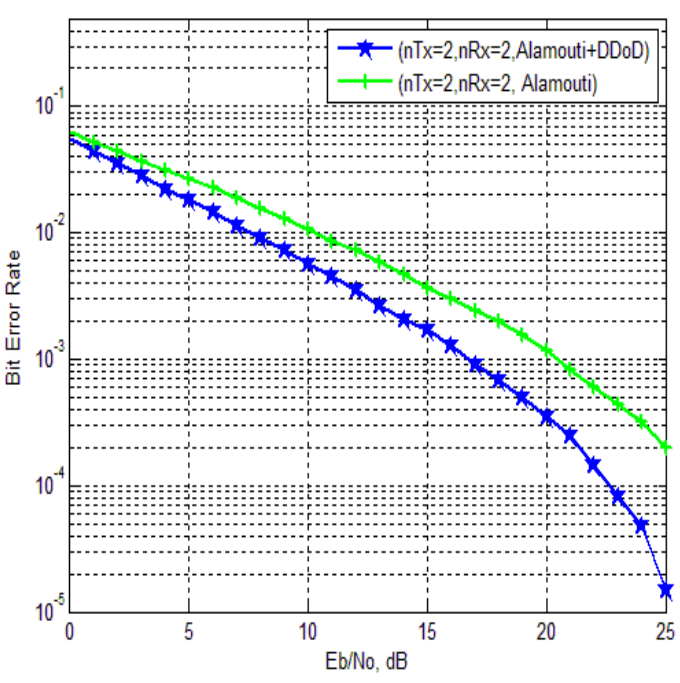

Fig. 10 The effect of discontinues DDoD

enhanced with DDoD and Alamouti standard. We used the $2 \mathrm{k}$ mode (2048 points IFFT) with 4-QAM modulation. The guard interval length is $N_{G}=1 / 4$. At the receiver sides, we assumed that the channel estimation was perfect. For our investigations, we used indoor commercial-channel B models (used in Fig.6). Compared to the Alamouti standard which required an SNR of 21 $\mathrm{dB}$ at $\mathrm{BER}=10 \mathrm{e}-3$, an Alamouti enhanced using DDoD requires $16.5 \mathrm{~dB}$, which provides a gain of $4.5 \mathrm{~dB}$ for the considered DVB-T2 parameter set (Fig. 10).

In the next experiment, we compared different systems based on multiple antenna techniques; Alamouti, DD, CDD with different $d i$ to the MISO systems $(2 \times 1)$, and DDoD in Rayleigh fading channel. The previous channel, which was constant over two adjacent OFDM carriers (non-highly selective fading), was applied for this experiment (Fig. 11).

All MISO methods are based on standard Alamouti and the combination of that with other simple diversity techniques was compared. After removing the $\mathrm{CP}$ at the receiver side, as long as TX-antenna certain delays, $d_{i}$, is equal to cyclic shifts, $d_{i}^{c y c}$, both CDD and DD schemes yield the same signal. In this simulation condition, the DD scheme performs the same as CDD. However, there is a fact to take into account that if the $\mathrm{CP}$ length is increased for the DD scheme, the amount of interference will be huge. [32]. The OFDM symbols of the DD signal partly overlap with the CP of the subsequent OFDM symbol at about delay which is a restriction in the choice of $d_{i}$. As a result, Figure 11 shows that the DDoD combination is better than the other methods.

We have set up the third strategy to verify the better performance of the proposed $\Delta \mathrm{h}$-Alamouti decoder using 
the CDD scheme and DDoD with respect to $\triangle \mathrm{h}$-Alamouti, which was introduced in [1]. we observe a comprehensive comparison between $\Delta \mathrm{h}$-Alamouti, efficient $\Delta \mathrm{h}$-Alamouti using $\mathrm{CDD}$, and $\Delta \mathrm{h}$-Alamouti using DDoD in Fig. 12 and 13. The performance is evaluated using simulations in different channels that include the presence of high selective fading. The last experiment is applied in two different channels, which are introduced in [1] (also, is based on [26]).
In the first type of channel, we witness that efficient $\Delta \mathrm{h}$ Alamouti using CDD and $\triangle \mathrm{h}$-Alamouti using DDoD have a better performance than $\Delta \mathrm{h}$-Alamouti. Because, in two methods based on DDoD and CDD, diversity is exploited without the need for additional complexity at the receiver. This a considerable advantage of these types of methods. Also, these two proposed methods are driven great benefit from efficient encoding and decoding, which is explained in previous sections. However, in Fig.13, the $\Delta$ h-Alamouti

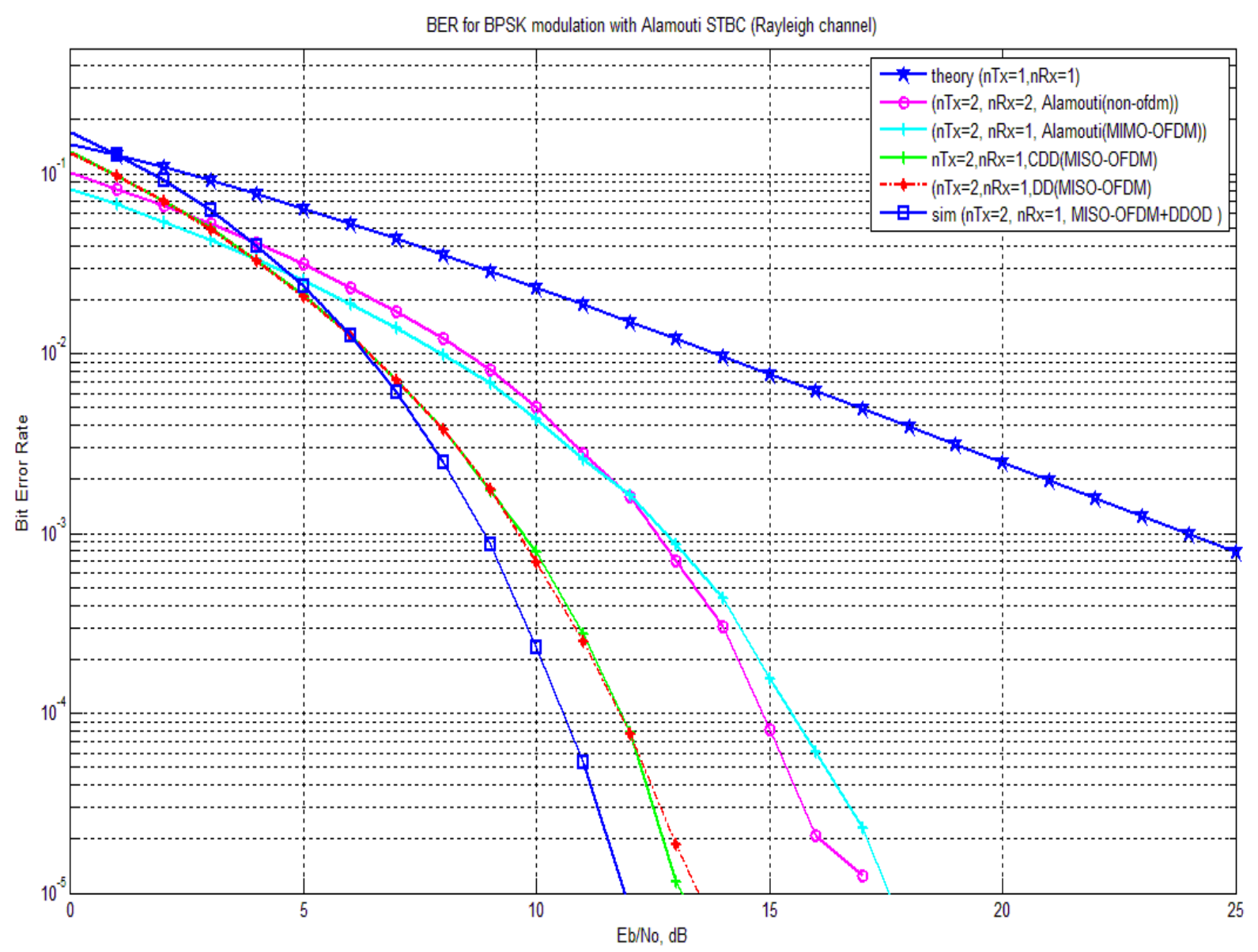

Fig. 11 A comparison of different schemes (theory, Alamouti, DD, CDD, and DDoD) 


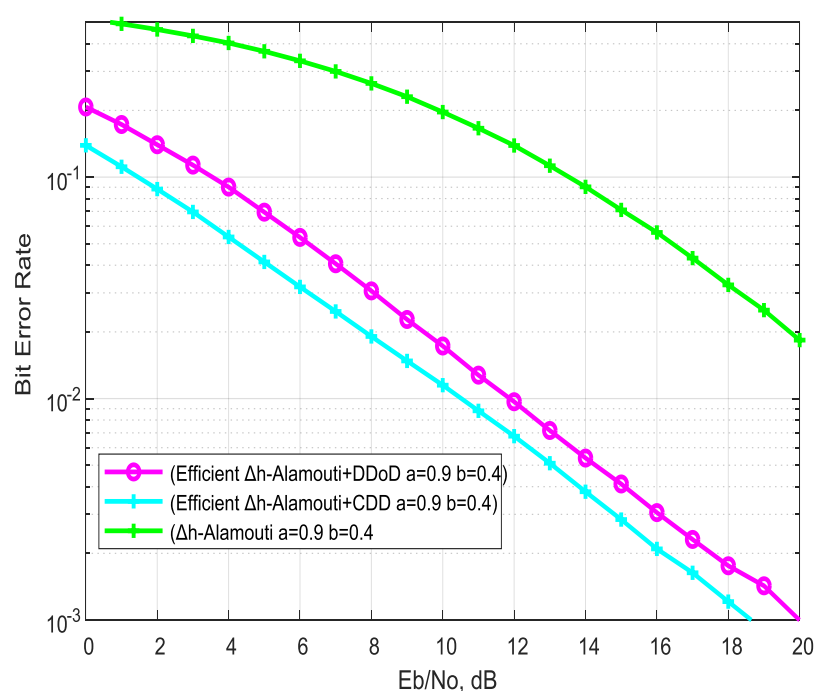

Fig. 12 A comparison of different schemes (Efficient $\Delta \mathrm{h}$ Alamouti,CDD, and DDoD for $\mathrm{a}=0.9, \mathrm{~b}=0.4$ )

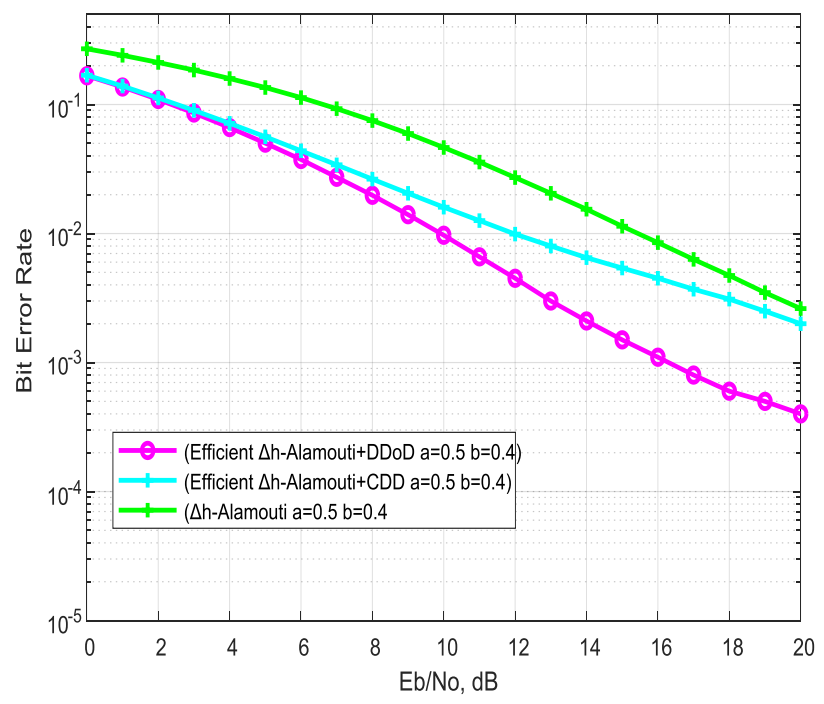

Fig. 13 A comparison of different schemes(Efficient $\Delta \mathrm{h}$ Alamouti,CDD, and DDoD for $\mathrm{a}=0.5, \mathrm{~b}=0.4$ )

\section{5- Conclusion}

In this paper, it was shown that the performance of wellknown Alamouti decoding degrades in the highly frequency selective channels. To tackle this problem, we proposed an efficient decoder using the CDD and DDoD scheme, which enhanced the standard Alamouti decoding and allowed high tolerability of the system in the frequency-selective channels by increasing the diversity.

Due to standard compatibility, a receiver does not need to be aware of their CDD and DDoD implementation. Also, the effect of the DDoD scheme is well noticeable on the receiver side. Analysis and simulation results demonstrated that the proposed scheme has significant performance improvement. Therefore, the new scheme is useful in MIMO-OFDM systems such as the DVB-T2 standard, which allows a high reliability and capacity enhancement. Due to all these features and as a future work, many wireless systems could take advantage of these schemes, explored with a low complexity implementation.

\section{References}

[1] A. Omri, R. Hamila, A. Hazmi, R. Bouallegue, and A. AlDweik, "Enhanced Alamouti decoding scheme for DVB-T2 systems in SFN channels," in 2011 IEEE 22nd International Symposium on Personal, Indoor and Mobile Radio Communications, 2011, pp. 1626-1630, DOI: 10.1109/PIMRC.6139779.

[2] E. P. Simon, L. Ros, H. Hijazi, and M. Ghogho, "Joint Carrier Frequency Offset and Channel Estimation for OFDM Systems via the EM Algorithm in the Presence of Very High Mobility," IEEE Trans. Signal Process, vol. 60, no. 2, pp. 754-765, Feb. 2012, DOI: 10.1109/tsp.2011.2174053.

[3] L. E. Nazarov, "Signal constructions based on OFDM signals resistant to the influence of spectrum-concentrated interference," J. Commun. Technol. Electron., vol. 64, no. 8, pp. 774-782, 2019.

[4] J. Yao et al., "Comprehensive study on MIMO-related interference management in WLANs," IEEE Commun. Surv. Tutor., vol. 21, no. 3, pp. 2087-2110, 2019. DOI: 10.1109/COMST.2019.2894160.

[5] K. Yang, N. Yang, C. Xing, J. Wu, and Z. Zhang, "Spacetime network coding with transmit antenna selection and maximal-ratio combining," IEEE Trans. Wirel. Commun., vol. 14, no. 4, pp. 2106-2117, 2015. DOI: 10.1109/TWC.2014.2381217.

[6] E. Biglieri, R. Calderbank, A. Constantinides, A. Goldsmith, A. Paulraj, and H. V. Poor, "Capacity limits of MIMO system," in MIMO Wireless Communications. Cambridge, England: Cambridge University Press, 2009.

[7] N. Fernando, Y. Hong, and E. Viterbo, "MIMO SelfHeterodyne OFDM," IEEE Trans. Veh. Technol., vol. 65, no. 3, pp. 1271-1280, 2016

[8] S. H. Ahmed, S. M. U. Talha, and A. Khan, "Performance evaluation of DVB-T based OFDM over wireless communication channels," Lect. Notes Eng. Comput. Sci., Vol. 2195, 2012.

[9] M. Tormos, C. Tanougast, A. Dandache, D. Masse, and P. Kasser, "Modeling and performance evaluations of Alamouti technique in a single frequency network for DVB-T2,” EURASIP J. Wirel. Commun. Netw., vol. 2013, no. $1,2013$.

[10] T. H. Liu, "Analysis of the Alamouti STBC MIMO System With Spatial Division Multiplexing Over the Rayleigh Fading Channel," IEEE Trans. Wirel. Commun., Vol. 14, no. 9, 2015, pp. 5156-5170, DOI: 10.1109/TWC.2015.2433924.

[11] A. Dammann and S. Kaiser, "Low Complex Standard Conformable Antenna Diversity Techniques for OFDM 
Systems and its Application to the DVB-T System,", ITG FACHBERICHT. 2002, pp. 253-60.

[12] N. Cornillet, M. Crussiere, and J. F. Helard, "Performance of the DVB-T2 system in a single frequency network: Analysis of the distributed alamouti scheme," in 2011 IEEE International Symposium on Broadband Multimedia Systems and Broadcasting (BMSB), 2011, DOI: 10.1109/BMSB.2011.5954961.

[13] K. Yang, N. Yang, C. Xing, J. Wu, and J. An, "SpaceTime Network Coding With Antenna Selection," IEEE Trans. Veh. Technol., Vol. 65, no. 7, 2016, pp. 5264-5274, DOI: 10.1109/TVT.2015.2455233.

[14] R. Zakaria and D. Le Ruyet, "A novel filter-bank multicarrier scheme to mitigate the intrinsic interference: Application to MIMO systems," IEEE Trans. Wirel. Commun., vol. 11, no. 3, pp. 1112-1123, 2012. DOI: 10.1109/TWC.2012.012412.110607.

[15] S. Lu, S. Member, B. Narasimhan, and S. Member, "A Novel SFBC-OFDM Scheme for Doubly-Selective Channels," IEEE Trans. Veh. Technol.,Vol. 58(5), pp. 2573-2578, 2008.

[16] K. A. Banawan and E. Sourour, "Turbo equalization of precoded collaborative MIMO for the uplink of LTEadvanced," presented at the 2013 International Conf. on Computing, Networking and Communications (ICNC 2013), Jan. 2013, DOI: 10.1109/ICCNC.2013.6504225.

[17] Y. Jung and H. Song, "Low-Complexity and Robust Symbol Timing Synchronization Scheme for MIMO DVBT2 Systems," IEEE Access, Vol. 6, 2018, pp. 4338443391, DOI: 10.1109/ACCESS.2018.2863255.

[18] T. Arbi, B. Geller, J. Yang, C. A. Nour, and O. Rioul, "Uniformly Projected RCQD QAM $\square$ : A Low-Complexity Signal Space Diversity Solution Over Fading Channels With or Without Erasures," IEEE Trans. Broadcast., Vol. 64, no. 4, 2018, pp. 803-815, DOI: 10.1109/TBC.2018.2811618.

[19] T. Sato, B. Roland, P. Ivan, and A. Pablo, "A comparison between theoretical and practical planning approaches for DVB-T2 single frequency networks," in 2015 IEEE International Symposium on Broadband Multimedia Systems and Broadcasting, 2015., pp. 1-6.

[20] L. Fay, L. Michael, D. Gomez-Barquero, N. Ammar, and M. W. Caldwell, "An overview of the ATSC 3.0 physical layer specification," IEEE Trans. On Broadcast., vol. 62, no. 1, pp. 159-171, 2016. pp. 159-171, DOI: 10.1109/TBC.2015.2505417.

[21] A. A. A. Solyman et al., "A low-complexity equalizer for video broadcasting in cyber-physical social systems through handheld mobile devices," IEEE Access, vol. 8, pp. 67591-67602, 2020. DOI: 10.1109/ACCESS.2020.2982001

[22] Y. H. You and J. H. Paik, "Suboptimal Maximum Likelihood Detection of Integer Carrier Frequency Offset for Digital Terrestrial Television Broadcasting System," IEEE Trans. Broadcast., Vol. 66, no. 1, 2020, pp. 195-202, DOI: 10.1109/TBC.2019.2921663.

[23] S. M. Alamouti, "A simple transmit diversity technique for wireless communication," IEEE J. Sel. Areas Commun., Vol. 16, no. 8, pp. 1451-1458, 1998.

[24] D. Gozálvez, D. Gómez-Barquero, D. Vargas, and N. Cardona, "Time diversity in mobile DVB-T2 systems,"
IEEE Trans. Broadcast., Vol. 57, no. 3, pp. 617-628, 2011, DOI: 10.1109/TBC.2011.2161189.

[25] S. Kaiser, "Spatial transmit diversity techniques for broadband OFDM systems," IEEE. Global Telecommunications Conf. (Cat. No. 00CH37137), 2000, Vol. 3, pp. 1824-1828).

[26] Y. Zhang, J. Cosmas, K. K. Loo, M. Bard, and R. D. Bari, "Analysis of cyclic delay diversity on DVB-H systems over spatially correlated channel," IEEE Trans. Broadcast., Vol. 53, no. 1, pp. 247-254, 2007, DOI: 10.1109/TBC.2007.891702.

[27] S. Plass, A. Dammann, G. Richter, and M. Bossert, "Channel correlation properties in OFDM by using timevarying cyclic delay diversity," J. Commun., Vol. 3, no. 3, pp. 19-26, 2008.

[28] M. Liu, M. Crussi, and A. M. T. Model, "Enhanced Mobile Digital Video Broadcasting with Distributed SpaceTime Coding," in 2012 IEEE International Conf. on Communications (ICC), 2012, pp. 6971-6976.

[29] S. Plass, A. Dammann, and S. Sand, "An overview of cyclic delay diversity and its applications," IEEE Veh. Technol. Conf., 2008, DOI: 10.1109/VETECF.2008.133.

[30] A. Dammann, "On the influence of cyclic delay diversity and doppler diversity on the channel characteristics in OFDM systems," IEEE Int. Conf. Commun., 2007, pp. 4179-4184, DOI: 10.1109/ICC.2007.689.

[31] R. Raulefs, A. Dammann, S. Kaiser, and G. Auer, "The Doppler Spread - Gaining Diversity for Future Mobile Radio Systems," IEEE Glob. Telecommun. Conf. (IEEE Cat. No.03CH37489), 2003, pp. 1301-1305.

[32] B. Akbarian, S. Ghazi.M, "Better Performance of New Generation of Digital Video Broadcasting-terrestrial ( DVB-T2 ) using Alamouti scheme with Cyclic Delay Diversity," JIST, vol. 10, 108-114, 2015.

[33] ITSI, Implementation guidelines for a second generation digital terrestrial television broadcasting system (DVB-T2), ETSI, no. February 2012.

[34] Y. Fang et al., "Improved passive SAR imaging with DVB-T transmissions," IEEE Trans. Geosci. Remote Sens., Vol. 58, no. 7, 2020, pp. 5066-5076, DOI: 10.1109/TGRS.2020.2972156.

[35] A. Dammann, R. Raulefs, and S. Plass, "Soft cyclic delay diversity and its performance for DVB-T in ricean channels," GLOBECOM - IEEE Glob. Telecommun. Conf., 2007, pp. 4210-4214, DOI: 10.1109/GLOCOM.2007.801.C

[36] Q. Hamarsheh and O. Daoud, "Enhancing DVB-H BER based OFDM systems," J. Commun. Technol. Electron., Vol. 60, no. 8, pp. 880-889, DOI: 10.1134/S1064226915080069, 2015.

[37] A. Dammann, S. Plass, and S. Sand, "Cyclic delay diversity - A simple, flexible and effective multi-antenna technology for OFDM," IEEE Int. Symp. Spread Spectr. Tech. Appl., 2008, pp. 550-554, DOI: 10.1109/ISSSTA.2008.108.

[38] C. Gómez-Calero, L. Cuéllar, L. De Haro, and R. Martínez, "A 2 x 2 novel MIMO testbed for DVB-T2 systems," 2009 IEEE Int. Symp. Broadband Multimed. Syst. Broadcast. BMSB 2009, 2009, DOI: 10.1109/ISBMSB.2009.5133823. 
[39] W. Zhou, J. Wu, and P. Fan, "High Mobility Wireless Communications with Doppler Diversity: Fundamental Performance Limits," IEEE Trans. Wirel. Commun., Vol. 14, no. 12, pp. 6981-6992 , 2015, DOI: 10.1109/TWC.2015.2463276.J

[40] L. De Haro, “A 2 x 2 MIMO DVB-T2 System $\square$ : Design, New Channel Estimation Scheme and Measurements With Polarization Diversity," IEEE Trans. On Broadcast, 56(2), Vol. 56, no. 2, pp. 184-192,2010.

[41] K. Chanthirasekaran, M. A. Bhagyaveni, and L. R. Parvathy, "Multi-Parameter Based Scheduling for Multiuser MIMO Systems," J. Electr. Eng. Technol Vol. 10, no. 6, pp. 2406-2412, 2015.

[42] C. W. Tan and A. R. Calderbank, "Multiuser detection of alamouti signals," IEEE trans. commun., vol. 57, no. 7, pp. 2080-2089, 2009, DOI: 10.1109/TCOMM.2009.07.070592.

[43] R. Chai, A.L. Savvaris, and A. Tsourdos, "Violation learning differential evolution-based hp-adaptive pseudospectral method for trajectory optimization of space maneuver vehicle," IEEE Trans. Aerosp. Electron. Syst., vol. 53, no. 4, pp. 2031-2044, 2017

[44] F. Qu, Z. Wang and L. Yang, "Differential orthogonal space-time block coding modulation for time-variant underwater acoustic channels," IEEE j. ocean. eng., pp. 111,2016

Behnam Akbarian received the B.S. degree in electrical engineering from Islamic Azad University in 2008 and the M.sc. degree in telecommunication systems from Islamic Azad University in 2013. He is a Ph.D. Candidate at Azad University. His main research interests include digital communications with an emphasis on Massive MIMO, New waveform for 5G, and antenna diversity techniques for broadcast systems and space-time coding.

Saeed Ghazi-Maghrebi is a assistant professor of communication Engineering department of Azad University, Yadegar-e-Imam Khomeini (RAH) Share-Rey Branch, Tehran, Iran. He was born in Iran in 1963. He received the B.S. degree in electrical engineering from Kerman University, Iran, in 1988, and the M.S. degree from the Khajeh Nasir-edin- toosi University of Technology, Iran, in 1995. He received the Ph.D. degree in digital communications from Islamic Azad University, Iran in 2010. His current research interests are digital communication, signal processing, and adaptive filtering. 